\title{
Article
}

\section{Simulation methods for system reliability using the survival signature}

Patelli, Edoardo, Feng, Geng, Coolen, Frank P.A. and Coolen-Maturi, Tahani

Available at http://clok.uclan.ac.uk/25622/

Patelli, Edoardo, Feng, Geng ORCID: 0000-0002-4705-3252, Coolen, Frank P.A. and Coolen-Maturi, Tahani (2017) Simulation methods for system reliability using the survival signature. Reliability Engineering \& System Safety, 167 . pp. 327-337. ISSN 0951-8320

It is advisable to refer to the publisher's version if you intend to cite from the work. http://dx.doi.org/10.1016/j.ress.2017.06.018

For more information about UCLan's research in this area go to http://www.uclan.ac.uk/researchgroups/ and search for <name of research Group>.

For information about Research generally at UCLan please go to http://www.uclan.ac.uk/research/

All outputs in CLoK are protected by Intellectual Property Rights law, including Copyright law. Copyright, IPR and Moral Rights for the works on this site are retained by the individual authors and/or other copyright owners. Terms and conditions for use of this material are defined in the policies page.

\section{CLoK}

Central Lancashire online Knowledge www.clok.uclan.ac.uk

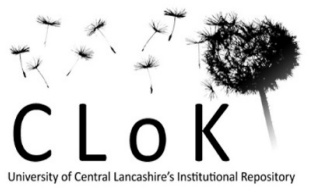




\title{
Simulation Methods for System Reliability Using the Survival Signature
}

\author{
Edoardo Patelli ${ }^{1, *}$, Geng Feng ${ }^{1}$, Frank P.A. Coolen ${ }^{2}$, Tahani Coolen-Maturi ${ }^{3}$ \\ ${ }^{1}$ Institute for Risk and Uncertainty, University of Liverpool, Liverpool, United Kingdom \\ ${ }^{2}$ Department of Mathematical Sciences, Durham University, Durham, United Kingdom \\ ${ }^{3}$ Durham University Business School, Durham University, Durham, United Kingdom
}

\begin{abstract}
Recently, the survival signature has been presented as a summary of the structure function which is sufficient for computation of common reliability metrics and has the crucial advantage that it can be applied to systems with components whose failure times are not exchangeable. The survival signature provides a huge reduction in required information, e.g. for its storage, compared to the full structure function, its implementation to larger systems is still difficult in a purely analytical manner and simulations may be required to derive the reliability metrics of interest. Hence, the main question addressed in this paper is whether or not the survival signature provides sufficient information for efficient simulation to derive the system's failure time distribution. We answer this question in the affirmative by presenting two algorithms for survival signature-based simulation. In addition, we present a third simulation algorithm that can be used in case of repairable components. It turns out that these algorithms are very efficient, beyond the initial advantage of requiring only the survival signature to be available, instead of the full structure function.
\end{abstract}

Keywords: Reliability Analysis; Survival Signature; Monte Carlo Method; Complex Systems; Multi state components.

\section{Introduction}

The study of the reliability of complex systems, particularly systems with a structure that cannot be sequentially reduced by considering alternative series and parallel subsystems, is a topic subject which has attracted much attention in

\footnotetext{
* Corresponding author

Email address: edoardo.patelli@liverpool.ac.uk (Edoardo Patelli ${ }^{1, *}$ )
} 
the literature and which is of obvious importance in many application areas [1]. Traditionally, the reliability analysis of systems is performed adopting different well-known tools such as reliability block diagrams, fault tree and success tree methods, failure mode and effect analysis, and master logic diagrams [2]. The main limitation of these traditional approaches for applicability to large complex systems is due to the complex and tedious calculations for finding minimal path sets and cut sets. For instance, for a system with $m$ components $2^{m}$ combinations of component states must be specified, which is impossible for most practical systems and networks. Instead, if the systems components can be divided into groups with exchangeable failure times, the survival signature is sufficient to derive the systems failure time distribution given the components failure time distributions.

In addition, when the information about the system is not perfect, for example leading to imprecise probabilities being required to quantify the uncertainties, it is even more difficult to apply those methods.

In recent years, the system signature has been recognized as a useful tool to quantify the reliability of systems consisting of independent and identically distributed (iid) or exchangeable components with respect to their random failure times $[3,4]$, we say that such systems only have 'components of one type'. The system signature enables full separation of the system structure from the component probabilistic failure time distribution when deriving the system failure time

\section{with more than one component type is not really possible as it requires the compu-} tation of the probabilities of different orderings of order statistics of the different failure time distributions involved [5], which tends to be intractable.

In order to overcome the limitations of the system signature, Coolen and Coolen-Maturi $[5,6]$ presented the survival signature, which has the same merits as the system signature for systems consisting of a single type of components, but it is also an effective tool for analysing complex systems consisting of multiple component types. Therefore, the survival signature is a useful tool for reliability quantification for complex systems and networks because it only needs to be calculated once providing a massive reduction of the computational cost required by the analysis. Recently, Coolen et al. [7] presented non-parametric predictive inference for system reliability using the survival signature, and Aslett et al. [8] did similarly within the Bayesian framework of statistics. Feng et al. [9] developed an analytical method to calculate the survival function of systems with uncertainty about pa40 rameters of assumed component failure time distributions. These methods are all useful, but may become less practical for larger complex systems. System survival signature can also be derived from subsystems' survival signatures, if these are in 
series-parallel configurations [7]. Recently, Reed presented and efficient computational approach for computing exact system and survival signatures of large and complex systems [10]. The survival signature together with the provided simulation algorithms provides a generalized tool for realistic quantification of system reliability.

Parameter uncertainties and imprecisions are generally epistemic in nature due to the lack of knowledge or data, or the unknown relationship between components (e.g., poor understanding of accident initiating events or coupled physics phenomena, lack of data to characterise experiment processes, random errors in measuring and analytic devices), all of them make it difficult to characterize probabilistically the failure time of components. Since the reliability and performance of systems are directly affected by uncertainties and imprecisions, a quantitative assessment of uncertainty is widely recognized as an important task in practical engineering $[11,12]$.

Simulation approaches are used to investigate large and complex systems and for obtaining numerical solutions where analytical solutions are not available. In particular, simulation methods allow to consider explicitly the effect of uncertainty and imprecision on the system under investigation providing a powerful tool for risk analysis which allows better decision making under uncertainty. Simulation method can be used to e.g. identify problems before implementation, evaluate ideas and identify inefficiencies, understand why observed events occur.

The use of simulation methods for system reliability has many attractive features. Generally, it can be used for the sensitivity analysis of multi-criteria decision model [13], optimize models with rare events [14] and perform multi-attribute decision making [15]. Most of the current simulation methods are based on Monte Carlo simulation and structure function. By generating the state evolution of each component, the structure function is computed to determine the state of the system. The structure function is in a boolean format and can only be used to identify a specific output of the system. More structure functions can be used to match all the possible status of the system at the cost of increasing the complexity of the analysis (see e.g. [16]). Several methods are available for the reliability analysis of complex system based on structural function (see e.g. $[17,4,18]$ ). However, we envisage a scenario, particularly for large systems, where the full system structure information (or structure function, min paths sets etc.) is not available but only the survival signature that represents a summary of the structure function. In particular, for very large scale systems and networks, storing only the survival signature and not the entire structure function is clearly advantageous.

In this paper, we will show that the survival signature is sufficient for basic 
reliability inferences (e.g. determining the system reliability function) which can be used for further inferences and decision support. Efficient simulation approaches are proposed to estimate the reliability of systems based on survival signature. The method is particularly useful when the probability term of the survival function (as shown later in the Eq. 2 representing the probability of the components working at specific time), cannot easily be derived analytically but the failure times for the exchangeable components can nevertheless be sampled through simulation.

The proposed simulation approaches are generally applicable to any system configuration. In addition, it allows to consider different representation of the uncertainties including system multi-state components (i.e. repairable components). The numerical implementation of the proposed approaches is based on two open source packages: the R package "ReliabilityTheory" [19, 20] adopted to calculate the survival signature and OpenCossan [21, 22] a Matlab toolbox for uncertainty quantification and reliability analysis used to simulate the system evolution. The applicability and efficiency of the proposed approaches are demonstrated by solving numerical examples.

This paper is organized as follows. Section 2 presents a brief overview of the survival signature and the related system survival function. Survival signaturebased simulation methods for system reliability are presented in Section 3. In Section 4, the applicability and performance of the proposed approaches is shown by analysing four numerical examples. Finally Section 5 closes the paper with conclusions.

\section{Survival Signature}

Suppose there is one system consisting of $m$ components. Let the state vector of components be $\underline{x}=\left(x_{1}, x_{2}, \ldots, x_{m}\right) \in\{0,1\}^{m}$ with $x_{i}=1$ if the $i$ th component is in working state and $x_{i}=0$ if not. $\phi=\phi(\underline{x}):\{0,1\}^{m} \rightarrow\{0,1\}$ defines the system structure function, i.e., the system status based on all possible $\underline{x} . \phi$ is 1 if the system functions for state vector $\underline{x}$ and 0 if not.

Now consider a system with $K \geq 2$ types of $M$ components, with $m_{k}$ indicating the number of components of each type and $\sum_{k=1}^{K} m_{k}=M$. It is assumed that the failure times of the components of the same type are independently and identically distributed (iid) or exchangeable. Note that this is usually understood as implying that the components are 'exchangeable', e.g. produced by the same manufacturer. However, the assumed exchangeability of their failure times also implies a similarity of the tasks the components of the same type perform in the system, e.g. if similar components function at different stress levels their failure time distributions are likely not to be the same and hence for the reliability analysis using the survival signature such components would be considered to be of different types. The 
components of the same type can be grouped together because of the random ordering of the components in the state vector, which leads to a state vector can be written as $\underline{x}=\left(\underline{x}^{1}, \underline{x}^{2}, \ldots, \underline{x}^{K}\right)$, with $\underline{x}^{k}=\left(x_{1}^{k}, x_{2}^{k}, \ldots, x_{m_{k}}^{k}\right)$ representing the states of the components of type $k$. Coolen and Coolen-Mature [5] introduced the survival signature for such a system, denoted by $\Phi\left(l_{1}, l_{2}, \ldots, l_{K}\right)$, with $l_{k}=0,1, \ldots, m_{k}$ for $k=1,2, \ldots, K$, which is defined to be the probability that the system functions given that $l_{k}$ of its $m_{k}$ components of type $k$ work, for each $k \in\{1,2, \ldots, K\}$. There are $\left(\begin{array}{c}m_{k} \\ l_{k}\end{array}\right)$ state vectors $\underline{x}^{k}$ with precisely $l_{k}$ components $x_{i}^{k}$ equal to 1 , so with $\sum_{i=1}^{m_{k}} x_{i}^{k}=l_{k}(k=1,2, \ldots, K)$, and $S_{l_{1}, l_{2}, \ldots, l_{K}}$ denote the set of all state vectors for the whole system.

Assume that the random failure times of components of the different types are fully independent, and in addition the components are exchangeable within the same component types, then the survival signature is equal to:

$$
\Phi\left(l_{1}, \ldots, l_{K}\right)=\left[\prod_{k=1}^{K}\left(\begin{array}{c}
m_{k} \\
l_{k}
\end{array}\right)^{-1}\right] \times \sum_{\underline{x} \in S_{l_{1}, l_{2}, \ldots, l_{K}}} \phi(\underline{x}),
$$

where $C_{k}(t) \in\left\{0,1, \ldots, m_{k}\right\}$ is the number of $k$ components working at time $t$. The survival function of the system with $K$ types of components can be expressed as [5]:

$$
P\left(T_{S}>t\right)=\sum_{l_{1}=0}^{m_{1}} \ldots \sum_{l_{K}=0}^{m_{K}} \Phi\left(l_{1}, \ldots, l_{K}\right) P\left(\bigcap_{k=1}^{K}\left\{C_{k}(t)=l_{k}\right\}\right)
$$

If one can assume that the components of the same type have a known $\mathrm{CDF}, F_{k}(t)$ for type $k$, and that the failure times of different component types are independent, then these expressions are simplified using [5]:

$$
P\left(\bigcap_{k=1}^{K}\left\{C_{k}(t)=l_{k}\right\}\right)=\prod_{k=1}^{K} P\left(C_{k}(t)=l_{k}\right)=\prod_{k=1}^{K}\left(\begin{array}{c}
m_{k} \\
l_{k}
\end{array}\right)\left[F_{k}(t)\right]^{m_{k}-l_{k}}\left[1-F_{k}(t)\right]^{l_{k}}
$$

Equation (2) separates the structure of the system from the failure time distribution of its components, which is the main advantage of the survival signature, which it shares with the system signature. The survival signature only needs to be calculated once for any system, which is similar to the system signature for systems with only single type of components. The survival signature is closely related with system signature. For a special case of a system with only one type $(K=1)$ of components, the survival signature and the system signature [3] are directly linked to each other through a simple equation, however, the latter cannot 
be easily generalized for systems with multiple types $(K \geq 2)$ of components [5].

This implies that all attractive properties of the system signature also hold for the method using the survival signature. The survival signature is easy to apply for systems with multiple types of components, and one could argue it is much easier to interpret than the system signature. In addition, the quite simple survival signature (in particular for large systems with only relatively few different component types) and its monotonicity for coherent systems provide clear advantages to work towards approximations of the system reliability metrics. This does not limit the applicability of the survival signature to non-coherent systems (for example, electric distribution network or part of the electronic equipment of safety features). In such cases, the analysis of system with imprecision in the component failure time requires a full "double loop" approach as detailed in Section 3.4 .

\section{Simulation Methods}

Exact analytical solution can be obtained from Eq. 2 and Eq. 3. However, analytical solutions are restricted to particular cases (e.g. system with component failure time following exponential distribution and not repairable components). Instead, simulation methods can be applied to study and analyse any systems without introducing simplifications or unjustified assumptions.

The survival signature presented in the previous section can be adopted in a Monte Carlo based simulation method to estimate the system reliability in a simple and efficient way. A possible system evolution is simulated by generating random events (i.e. the random transition such as failure times of the system components) and then estimating the status of the system based on the survival signature (Equation 3). Then, counting the occurrence number of a specific condition (e.g. counting how many times the system is in working status), it is possible to estimate the reliability of the system. In this section, three Monte Carlo simulation methods adopting the survival signature are presented. The Algorithms 1 and 2 are used to estimate the reliability of non-repairable systems while Algorithm 3 can be applied for repairable systems and multi-state components as well.

\subsection{Algorithm 1}

The first simulation method is based on the realizations of failure events of the system's components. For each failure event the status of the system is generated based on the probability that the system is working knowing that a specific number of components are working. Such probability is given by the survival signature as defined in Equation (1). The survival signature is computed only once before starting the Monte Carlo simulation for instance using the approach presented in [10]. Suppose there is a system with $C$ components, $K$ component types and $m_{k}$ 
components of type $k$. Hence, $C=\sum_{k=1}^{K} m_{k}$. We assume that components of type $k$ have the same failure time distribution and that there is no repair opportunity for the components. The reliability of the system can be estimated adopting the following procedure:

Step 0. Initialise variables and counters (i.e. $V r$ );

Step 1. Sample the failure times for each component, $f_{i}$, for $i=1,2, \ldots, C$. The failure time of a component of type $k$ is obtained by sampling from the corresponding CDF $F_{k}$;

Step 2. Order the sequence of failure times $t_{i} \leq t_{i+1}$ for $i=1,2, \ldots, M$. Hence, $t_{1}$ represents the first failure of a system component, $t_{2}$ represents the second failure and so on;

Step 3. At each failure time, it is easy to calculate the number of components working for each component type: $C_{k}\left(t_{i}\right)$;

Step 4. Evaluate the survival signature which applies immediately after the corresponding failure indicated as $\Phi_{t_{i}} \equiv \Phi\left(C_{1}\left(t_{i}\right), C_{2}\left(t_{i}\right), \ldots, C_{K}\left(t_{i}\right)\right)$;

Step 5. Drawn from a Bernoulli distribution with probability $1-\Phi_{t_{1}}$ the system status $X_{1}$ at time $t_{1}$, if $X_{1}=1$ the system fails;

Step 6. If the system does not fail at $t_{1}$, then consider $t_{2}$. The probability that the system functions at time $t_{2}$ is $\Phi_{t_{2}} / \Phi_{t_{1}}=q_{2}$, given that it has survived at time $t_{1}$. So the system failure at time $t_{2}, X_{2}$, is drawn from a Bernoulli distribution with the probability $1-q_{2}$;

Step 7. Repeat Step 6 to process other failure times: Set $i=i+1$.

Step 8. Store the status of the system over the time, as follows: $\operatorname{Vr}(j)=\operatorname{Vr}(j)+$ $1 \forall j: j \cdot d t<t_{f}$ where $t_{f}$ is the system failure time and $d t$ represents the discretisation time.

The above procedure is repeated for $N$ samples and the estimate of the survival

function is obtained by averaging the vector collecting the status of the system over the number samples: $P\left(T_{s}>t\right) \approx \frac{V r(t)}{N}$.

This method simulates one system failure time in each run (Steps 1-7). It should be noted that with the assumption that the system fails if no component functions, this implies that there is an $i^{*}$, less than or equal to $C$, such that $q_{i^{*}}=0$. Hence the system fails certainly at this $t_{i^{*}}$ if it has not failed before.

A pseudo-algorithms of the simulation method is shown in Algorithm 1.

\subsection{Algorithm 2}

It is possible to estimate the system reliability without the necessity to sample the system status at each component failure time. The idea is to interpret the survival signature as a normalised "production capability" of the system defined 
by the Equation (1). For instance, if all the components are working, the system output is 1 . If all components are in failure status, the system output is 0 . Hence, instead of sampling the system state at each failure time, the survival signature is evaluated to collect the "production level of the system", i.e. the survival signature is evaluated immediately after each sampled component failure time and collected in proper counters. This can be obtained adopting the Algorithm 2 derived from the approach proposed by one of the authors used to estimated the production availability of an offshore installation requiring the derivation of the complete status of the system (based on the structural function and cut-sets) [16]. Here, a novel algorithm is proposed to estimate the reliability adopting the survival signature and hence avoiding the tedious calculation of all the system status.

The reliability of the system can be estimated modifying the Steps 5-7 of the Algorithm 1 as follows:

Step 5'. Compute the production level of the system by evaluating the survival signature at each time of interest $\Phi_{t_{i}}$. The probability that the system survives time $t_{1}$ is $\Phi_{t_{1}}$;

Step 6'. Collect the value of the survival signature in the vector $V r$ representing the survival function as follows: $\operatorname{Vr}(j)=\operatorname{Vr}(j)+\Phi_{t_{i}} \quad \forall j: j \cdot d t<t_{i}$ where $d t$ represents the discretization time.

The above procedure is repeated for $N$ samples and the reliability of the system is computed by averaging the values of the survival signature: $P\left(T_{s}>t\right) \approx \frac{1}{N} \operatorname{Vr}(t)$. The uses of the survival signature makes this approach extremely efficient since it does not require to sample the system output at each component transition time (i.e. component failures). For each Monte Carlo simulation, this method generates a random grid of time points at which to evaluate the survival signature representing the survival probability of the system at those times. Finally, the survival function is obtained by directly averaging the survival signature over the time.

A pseudo-algorithms of the simulation method for non-repairable components is shown in Algorithm 2 and the flow chart of the simulation methods proposed for estimate the reliability of non-repairable systems is shown in Figure 1a.

Algorithm 2 follows the productivity idea, which gives each run a possible survival function while Algorithm 1 gives a single system failure time in each run. Therefore, Algorithm 1 is useful for inference where one explicitly wants the simulated system failure times, whilst Algorithm 2 is efficient for inference on the system survival function.

It can be shown that the variance of the survival function estimator at each 


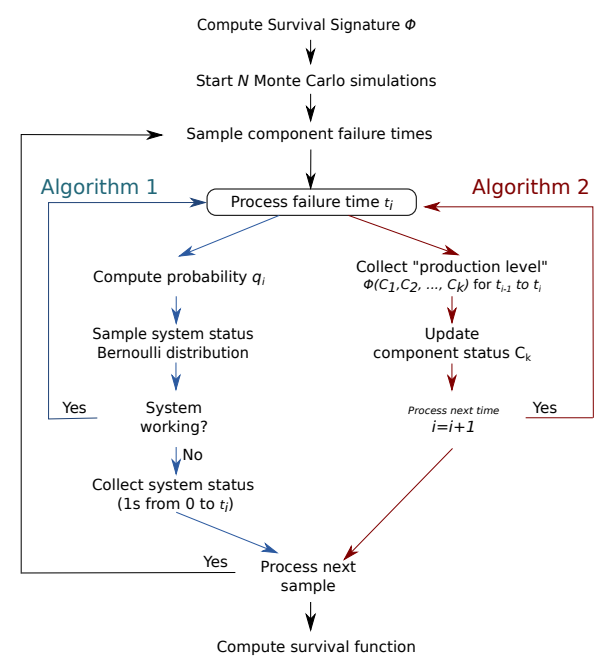

(a) Flow Chart of the Algorithms 1-2.

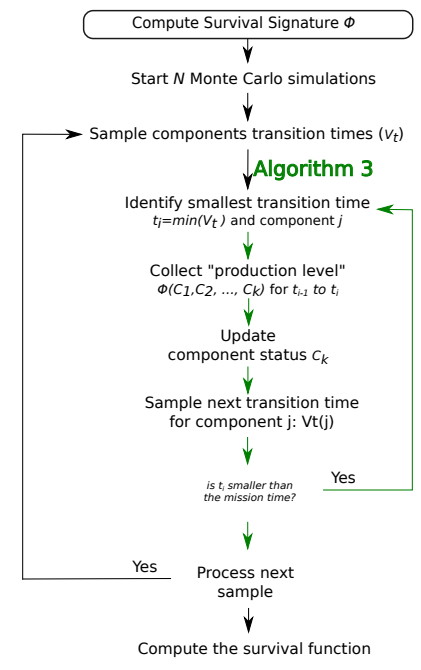

(b) Flow Chart of the Algorithm 3.

Figure 1: Flow Chart of the proposed algorithms.

time of interest obeys to the following formula [23]:

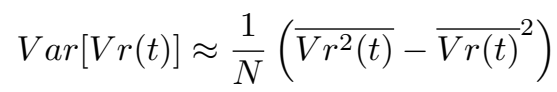

where $N$ represents the number of samples and $\overline{V r^{2}(t)}$ the mean of the square values of the survival function at time $t$ and $\overline{V r(t)}^{2}$ the square of the mean values of the survival function at time $t$. Also, in Equation (4) it is common to substitute $N-1$ in place of $N$ although the correction is negligible because $N \gg 1$. The Algorithm 2 tends to lead to better estimates of the system reliability when compared to Algorithm 1, as detailed in Section 4.1 and shown in Figure 5.

\subsection{Algorithm 3}

Algorithm 2 can easily be extended to analysing systems with multi-state components such as repairable systems. Assume that there are $j_{k}$ possible transitions for the components of type $k$. The probability of going from state $s=l$ to state $s^{\prime}=m$ in given by $p_{k l m}=\operatorname{Pr}\left(X_{k}=m \mid X_{k}=l\right)$. Let $F_{k l}=\sum_{m} \operatorname{Pr}\left(X_{k}=m \mid\right.$ $\left.X_{k}=l\right)=\operatorname{Pr}\left(\cdot \mid X_{k}=;\right)$ represents the CDF of the component of type $k$ to exit from its state $l$, i.e. to undergo a transition leading to a state $m \neq l$.

Let assume for the moment that there is only one possible transition to exit from the state $s=l$. For instance, a component in working status $s=1$ can fail and entering in the state $s^{\prime}=2$; the component in the state $s=2$ can only be repaired and returning in the status $s^{\prime}=1$. Hence, $p_{k 21}=\operatorname{Pr}\left(X_{k}=2 \mid X_{k}=1\right)=p_{k 2}$ 
represents the probability of failure for component $k, p_{k 12}=p_{k 1}$ the probability of repair. The Monte Carlo simulation is performed as follows.

Step 0. Initialise variables (i.e. $t_{\text {old }}=0$ ) and counters (i.e. $V t$ );

Step 1. Sample the transition times $t_{i}$ for $i=1,2, \ldots, C$ for each component of the system from the corresponding CDF, $F_{k l}$, and stored in a vector $V t=\left\{t_{1}, t_{2}, \ldots, t_{M}\right\}$, set $t_{\text {old }}=0 ;$

Step 2. Identify the first transition time, i.e. $\min (V t)$ and the corresponding component $z$. Hence, $t_{1}$ represents the first transition of the system, $t_{2}$ the second transition and so on;

Step 3. At each transition time $t_{i}$, calculate the number of components in working status (i.e. $C_{t_{i}}=\left(C_{1}, C_{2}, \ldots, C_{K}\right)$ ). The corresponding "production level" $\Phi_{t_{i}}$ is obtained by evaluating the survival signature for the number of components in working status;

Step 4. Collect the value of the survival signature at time $t_{i}, \Phi_{t_{i}}$, in a counter $V r$ representing the survival function as follows: $\operatorname{Vr}(j)=\operatorname{Vr}(j)+\Phi_{t_{i}} \quad \forall j$ : $t_{\text {old }} \leq j \cdot d t<\min (V t)$.

Step 5. Set $t_{\text {old }}=\min (V t)$ and sample the new status of the component $z$ from the probability mass function $P(s=m)=\frac{F_{k l m}\left(t_{i}\right)}{F_{k l}\left(t_{i}\right)}$;

Step 6. Update the vector of transition time $V t$ by sampling the next transition time $t_{z}^{\prime}$ of the component $z$ of type $k$ in status $m$ from $F_{k m}$. Hence: $V t(z)=t_{z}+t_{z}^{\prime}$

Step 7. If $\min (V t)<T_{F}$ (i.e. the final time), return to point 2 .

The above steps are repeated for $N$ samples and the survival function obtained by averaging the vector $V r$ over the number of samples. The flow chart of the proposed algorithm is shown in Figure $1 \mathrm{~b}$ and the pseudo-code is shown in Algorithm 3.

3.4. Reliability analysis of systems with imprecision

Reliability analysis of complex systems requires the probabilistic characterization of all the possible component transitions. This usually requires a large data-set that is not always available. In fact, it might not be possible to unequivocally characterize some component transitions due to lack of data or ambiguity. To avoid the inclusion of subjective knowledge or experts opinions, the imprecision and vagueness of the data can be treated by using concepts of imprecise probabilities.

Imprecise probability combines probabilistic and set theoretical components in a unified construct (see e.g. $[24,25,26]$ ). It allows a rational treatment of the information of possibly different forms without ignoring significant information, and without introducing unwarranted assumptions. For instance, if only few 
data points are available it might be difficult to identify the parameters and the form of a distribution [27]. An unknown value of a (deterministic) parameter is often modelled using a uniform distribution based on the principle of maximum entropy should be model as interval and not as distribution [28, 29]. In the analysis, imprecise probabilities combine, without mixing, randomness and imprecision. Randomness and imprecisions are considered simultaneously but viewed separately at any time during the analysis and in the results. The probabilistic analysis is carried out conditional on the elements from the sets, which leads eventually to sets of probabilistic results, see e.g. [30, 31, 32, 33]).

Considering the imprecision in the component parameters will lead to bounds of survival function of the systems and it can therefore be seen as a conservative analysis, in the sense that it does not make any additional hypothesis with regard to the available information. In some instances analytical methods will not be appropriate means to analyse a system. Again, simulation methods based on survival signature can be adopted to study systems considering parameter imprecision. A naive approach consists in adopting a double loop sampling where the outer loop is used to sample realization in the epistemic space. In other words, each realization in the epistemic space defines a new probabilistic model that needs to be solved adopting the simulation methods proposed above. Then the envelop of the system reliability is identified. However, since almost all the systems are coherent (system is coherent if each component is relevant, and the structure function is non decreasing), it is only necessary to compute the system reliability twice, using the lower and upper bounds for all the parameters, respectively. As shown in Refs. [7] and [9] assuming the components can not be repaired or replaced, the lower bound of the survival function can be computed as follows:

$$
\underline{S}_{T_{S}}(t)=\underline{P}\left(T_{S}>t\right)=\sum_{l_{1}=0}^{m_{1}} \ldots \sum_{l_{K}=0}^{m_{K}} \Phi\left(l_{1}, \ldots, l_{K}\right) \prod_{k=1}^{K} \bar{D}\left(C_{k}(t)=l_{k}\right)
$$

where $C_{k}(t)$ denotes the number of $k$ components working at time $t$, and

$$
\bar{D}\left(C_{k}(t)=l_{k}\right)=\bar{P}\left(C_{k}(t) \leq l_{k}\right)-\bar{P}\left(C_{k}(t) \leq l_{k}-1\right) .
$$

While the corresponding upper bound of the survival function is:

$$
\bar{S}_{T_{S}}(t)=\bar{P}\left(T_{S}>t\right)=\sum_{l_{1}=0}^{m_{1}} \ldots \sum_{l_{k}=0}^{m_{K}} \Phi\left(l_{1}, \ldots, l_{K}\right) \prod_{k=1}^{K} \underline{D}\left(C_{k}(t)=l_{k}\right)
$$




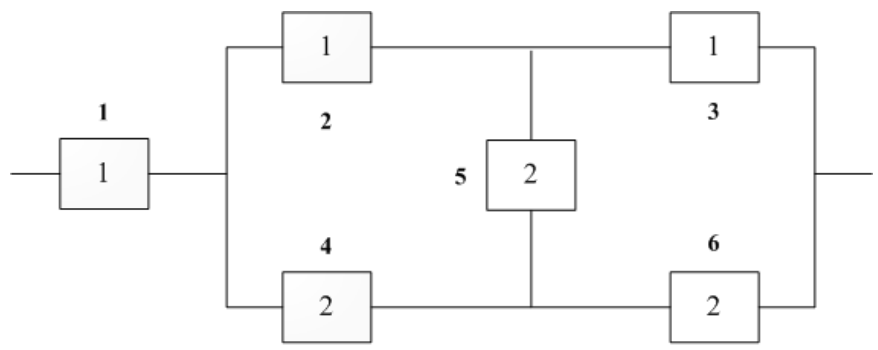

Figure 2: Bridge system with two types of components. The numbers inside the boxes indicate the component type. The numbers above the boxes indicate the component indices.

Table 1: Survival signature of the bridge system of Figure 2

\begin{tabular}{lll}
\hline$l_{1}$ & $l_{2}$ & $\Phi\left(l_{1}, l_{2}\right)$ \\
\hline 0 & {$[0,1,2,3]$} & 0 \\
{$[1,2]$} & {$[0,1]$} & 0 \\
1 & 2 & $1 / 9$ \\
1 & 3 & $1 / 3$ \\
2 & 2 & $4 / 9$ \\
2 & 3 & $2 / 3$ \\
3 & {$[0,1,2,3]$} & 1 \\
\hline
\end{tabular}

where

$$
\underline{D}\left(C_{k}(t)=l_{k}\right)=\underline{P}\left(C_{k}(t) \leq l_{k}\right)-\underline{P}\left(C_{k}(t) \leq l_{k}-1\right) .
$$

\section{Numerical Examples}

\subsection{Bridge system with not repairable components}

The purpose of this numerical example is to verify the proposed algorithms since for this simple problem analytical solutions are available. The system configuration is represented in Figure $2, k=1,2$. The bridge system comprises of six components, which belonging to two types. It has no series section or parallel section which can enable simplification. The survival signature can easily be computed either manually or using the R-package ReliabilityTheory [19]. The values of the survival signature are reported in Table 1 where $l_{1}$ and $l_{2}$ indicate the number of working component of type $k=1$ and $k=2$, respectively and $\Phi\left(l_{1}, l_{2}\right)$ is the survival signature of the bridge system. In this example the failure times of both component type 1 and 2 are obeying to exponential distributions with parameters $\lambda_{1}=0.8$ and $\lambda_{2}=1.5$, respectively, i.e. the components have a constant mean time to fail. It is also assumed that the component once failed can not be repaired.

The survival function of the bridge system is then calculated by means of the Algorithms 1 and 2. The resulting functions are then compared with the analytical 


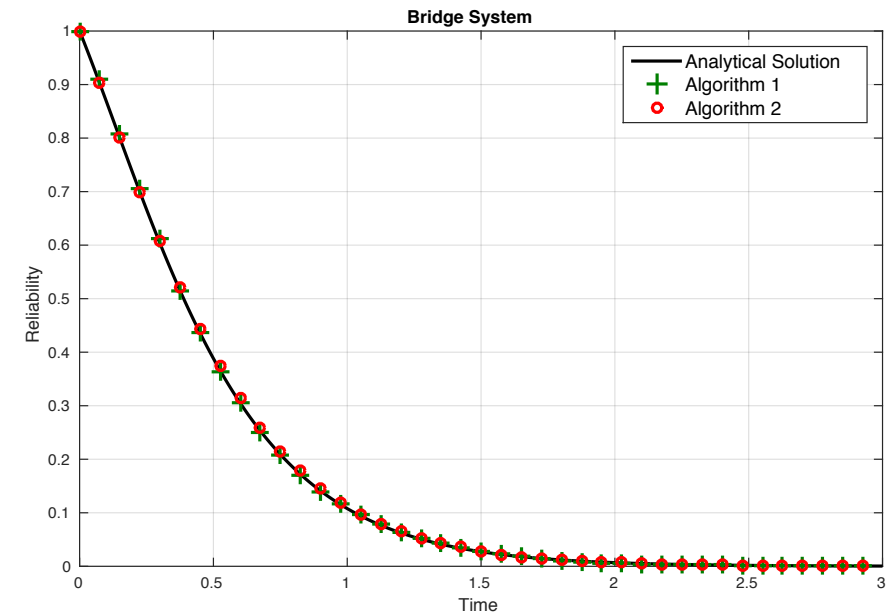

Figure 3: Survival function of the bridge system calculated by two simulation methods and analytical method, respectively.

solution. The survival function can be obtained from Equations (3) and (2):

$$
\begin{aligned}
P\left(T_{S}>t\right)= & \sum_{l_{1}=0}^{3} \sum_{l_{2}=0}^{3} \Phi\left(l_{1}, l_{2}\right)\left(\begin{array}{c}
3 \\
l_{1}
\end{array}\right)\left[1-e^{-0.8 t}\right]^{3-l_{1}}\left[e^{-0.8 t}\right]^{l_{1}} \times \\
& \left(\begin{array}{c}
3 \\
l_{2}
\end{array}\right)\left[1-e^{-1.5 t}\right]^{3-l_{2}}\left[e^{-1.5 t}\right]^{l_{2}}
\end{aligned}
$$

The results of the reliability analysis are shown in Figure 3, which shows a perfect agreement of the simulation methods with the analytical solution. The Monte Carlo simulation has been performed using $N=5000$ samples and a discretisation time $d t=0.0015$. The discretization time is only required to collect the numerical results (i.e. survival function) although the simulation of the system is continuous with respect to the time. Figure 4 shows an example of system evolution as a function of time with associate number of working component $C_{k}$. In order to show the efficiency of the proposed algorithm, the evolution of the variance of the estimators as a function of number of samples has been computed and shown in Figure 5. Algorithm 2 shows a smaller variance compared to Algorithm 1, in particular when small sample sizes are used.

The bridge system is also analysed in presence of imprecision on the parameters of the failure distribution time. In this case it is assumed that the parameters of the component failure time are not known precisely. The bounds of the parameter distributions are $\lambda_{1}=[0.4,1.2]$ and $\lambda_{2}=[1.3,2.1]$. The bounds of the survival functions are computed by means of the Algorithms 1 and 2. Since this system 


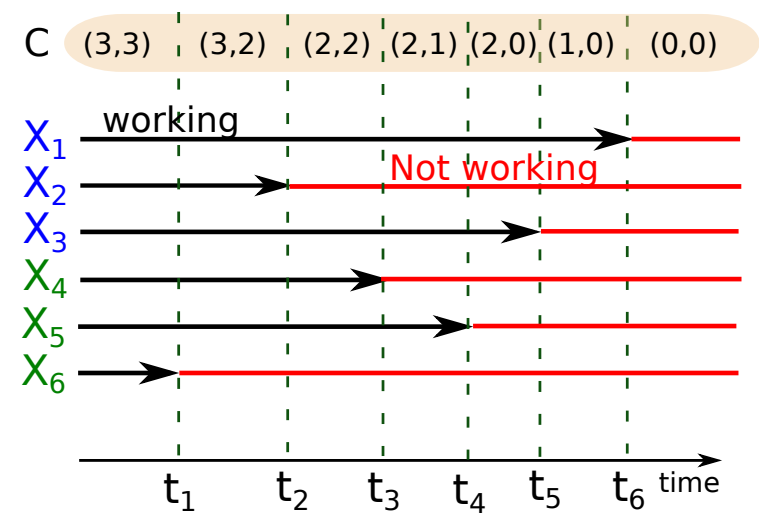

Figure 4: Realization of the number of working component $C_{k}$ as a function of time.

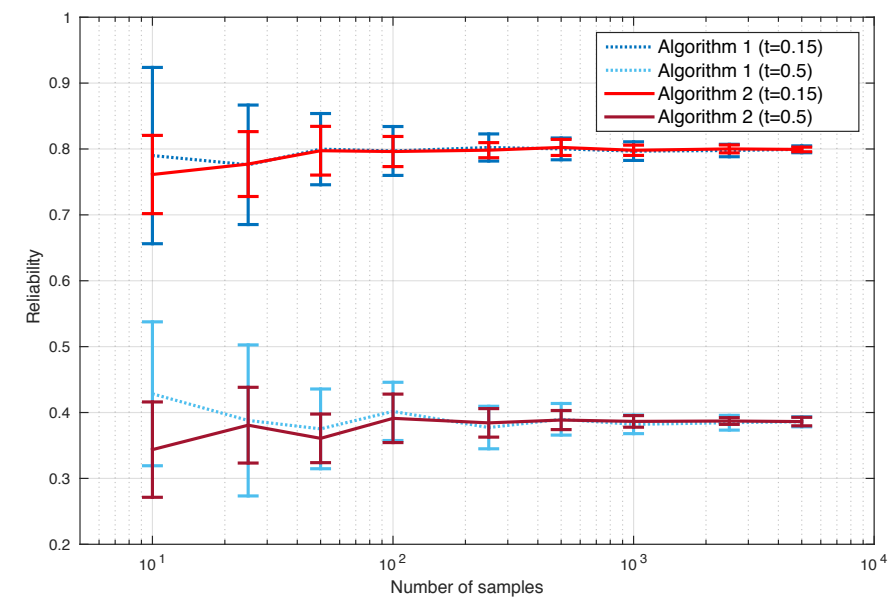

Figure 5: Variance of the estimator of the survival function.

is a coherent system, only two numerical simulations are required, i.e. the lower bound of the survival function is computed using the lower bounds of the parameter distribution and the upper bound is obtained adopting the upper bounds of the paramters $\lambda_{1}$ and $\lambda_{2}$. The results are then compared to the analytical solution adopting the method presented in [9]. The results of the simulation have been further verified by estimating the survival function adopting a double loop approach. The double loop sampling involves two layers of sampling: the outer loop, called the parameter loop, samples values from the set of distribution parameters; while the inner loop computes the survival function stating for the system knowing the precise probability distribution functions. Then, the lower and upper bounds of the survival function have been computed at each time of interest. The double loop Monte Carlo analysis has been performed using $N=5000$ samples for the 


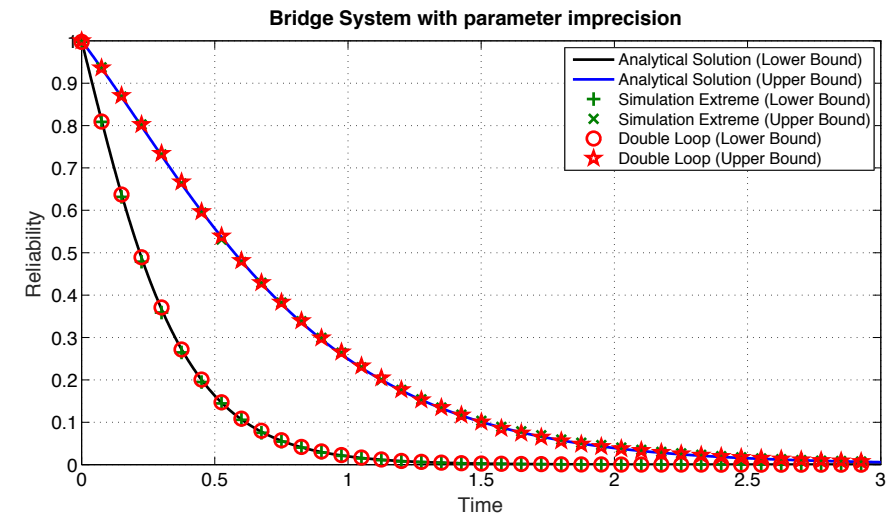

Figure 6: Bounds of the survival function of the bridge system calculated by means of the Algorithm 1 and 2 using bounds of the distribution parameter (Simulation Extreme) and compared with analytical solutions and the double loop approach.

inner loop and 1000 samples for the parameter loop. The results are collected in a counter using a discretisation time step $d t=0.0015$.

The results of the simulation considering imprecision are reported in Figure 6 showing a perfect agreement with the analytical solutions.

\subsection{Bridge system with repairable components}

In this example the components of the bridge system shown in Figure 2 are considered repairable. Hence, the components can be in two different states: working $(s=1)$ and not-working $(s=2)$. Two cases are analysed considering different distributions for the repair times as shown in Table 2. Analytical solutions are

Table 2: Parameters of repairable components in the bridge system. State 1: Working, State 2: Not-working.

\begin{tabular}{llll}
\hline C. type $(\mathrm{k})$ & Transition $(\mathrm{s})$ & Distribution & Parameters \\
\hline & \multicolumn{3}{c}{ CASE A } \\
\hline 1 & $1 \rightarrow 2$ & Exponential $(\lambda)$ & $(0.8)$ \\
2 & $2 \rightarrow 1$ & Weibull (scale,shape) & $(0.9,1.2)$ \\
2 & $1 \rightarrow 2$ & Exponential $(\lambda)$ & $(1.5)$ \\
& $2 \rightarrow 1$ & Weibull (scale,shape) & $(1.3,1.8)$ \\
\hline 1 & $1 \rightarrow 2$ & CASE B \\
1 & $2 \rightarrow 1$ & Exponential $(\lambda)$ & $(0.8)$ \\
2 & $1 \rightarrow 2$ & Uniform (min,max) & $(0.2,0.6)$ \\
2 & $2 \rightarrow 1$ & Exponential $(\lambda)$ & $(1.5)$ \\
& & Uniform (min,max) & $(0.1,0.2)$ \\
\hline
\end{tabular}

not available for analysing repairable systems and the system can only be analysed by adopting simulation methods such as the Algorithm 3. The estimated survival function $P\left(T_{S}>t\right)$ is shown in Figures 8 and 10 for the CASE A and B, respec- 
tively. An example of the evolution of the system is represented in Figure 7. The survival function reach a stationary level that depends on the ratio between the mean failure time and mean repair time.

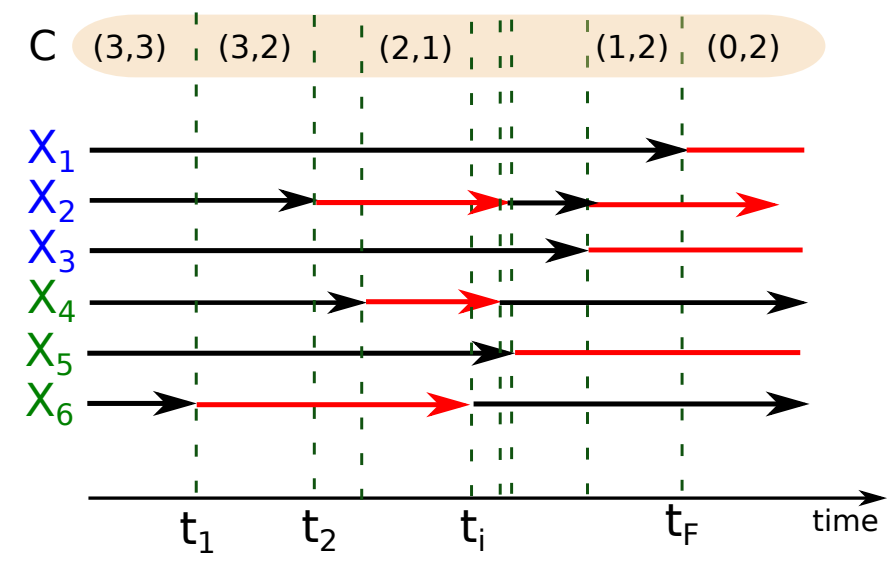

Figure 7: Realization of the number of working component $C_{k}$ as a function of time.

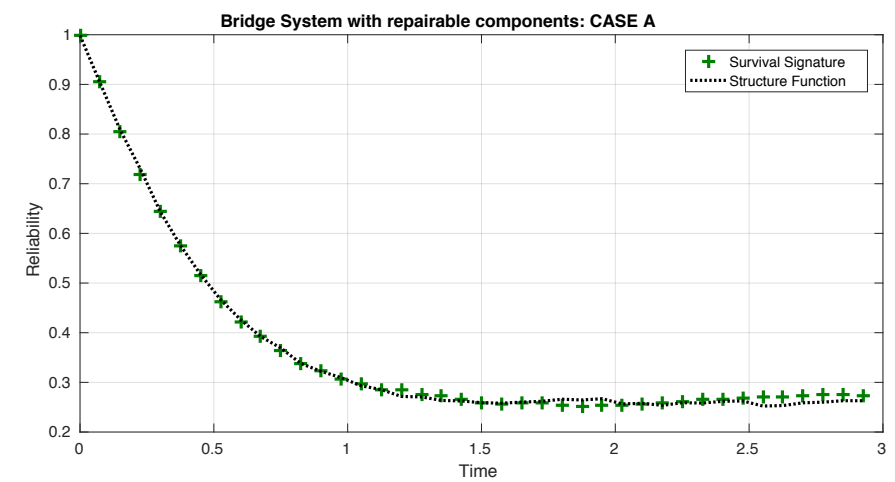

Figure 8: CASE A: Survival function of the bridge system with repairable components calculated by means of the Algorithm 3 and a simulation method based on structure function.

It is important to notice that the proposed approach (Algorithm 3) does not require the introduction of additional component types to analyse a system with repairable components. In order to verify the correctness of Algorithm 3 which is based on survival signature, the results have been compared with the solution of simulation method based on the structural function. The minimum path sets of the Bridge system shown in Figure 2 are $[1,2,3],[1,2,5,6],[1,3,4,5]$ and $[1,4,6]$. $N=5000$ samples have been used to estimate the reliability of the system and the results shown in Figures 8 and 10 are in perfect agreement with the results obtained using Algorithm 3. Figures 9 and 11 compare the variance of the estimator as a 


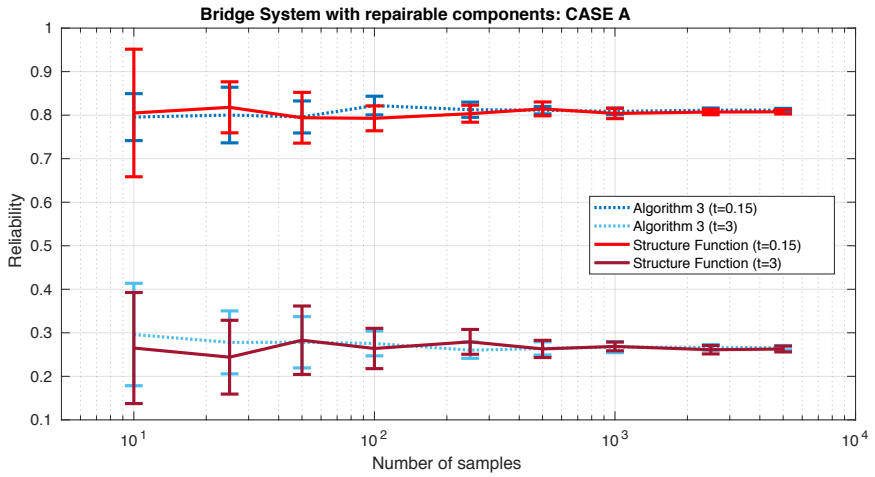

Figure 9: CASE A: Variance of the estimator for the bridge system with repairable components calculated by means of the Algorithm 3 and a simulation method based on structure function.

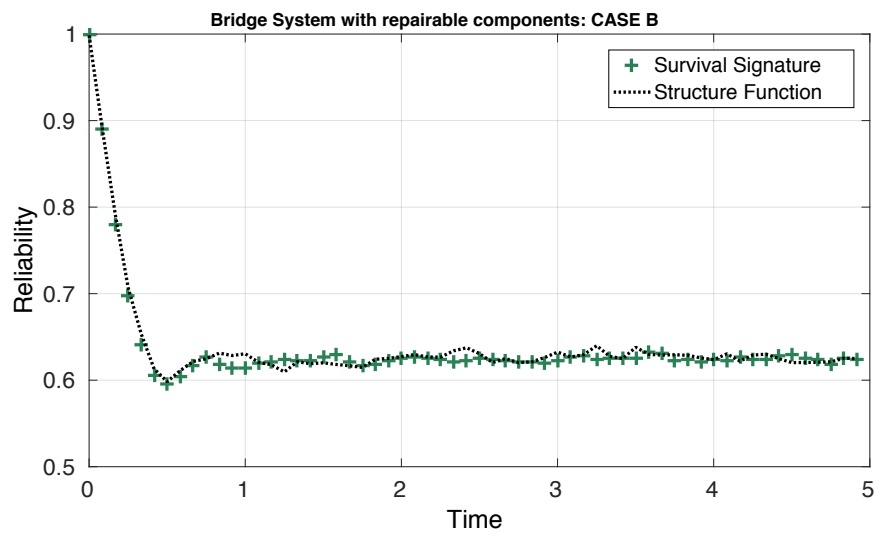

Figure 10: CASE B: Survival function of the bridge system with repairable components calculated by means of the Algorithm 3 and a simulation method based on structure function.

function of the number of samples adopting the Algorithm 3 based on survival signature and Monte Carlo method based on structural function.

\subsection{Grey System}

In order to illustrate the efficiency and the applicability of the proposed simulation approaches a complex system composed by 8 components of 5 types is analysed. The component failure types and distribution parameters are shown in Table 3, again affected by imprecision. In addition, it is assumed that the exact configuration of part of the system is unknown as shown in Figure 12, i.e. it might be composed by an additional component of type 1 or two components of type 2 connected in parallel. However, the system can still be described using the survival signature although affected by imprecision [6]. This has the advantages of more realistic reflections of uncertainty on system functioning and the proposed 


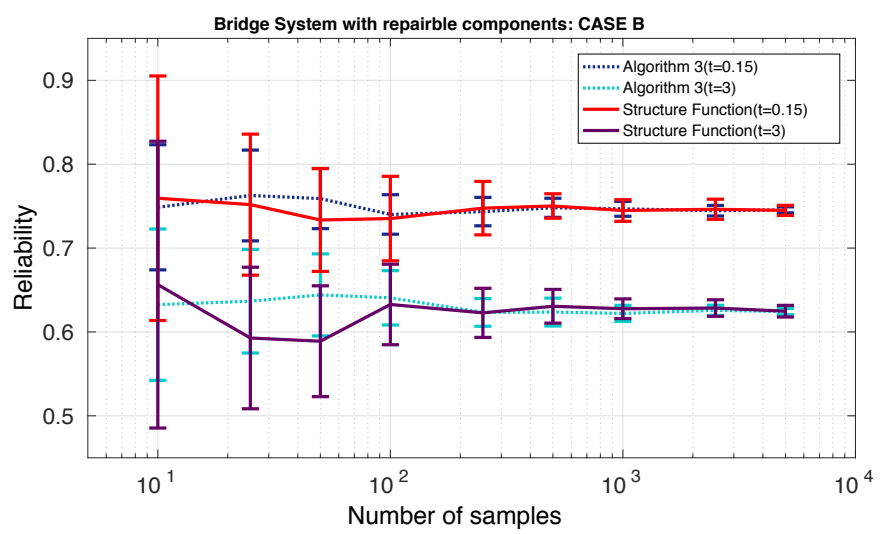

Figure 11: CASE B: Variance of the estimator with repairable components calculated by means of the Algorithm 3 and a simulation method based on structure function.

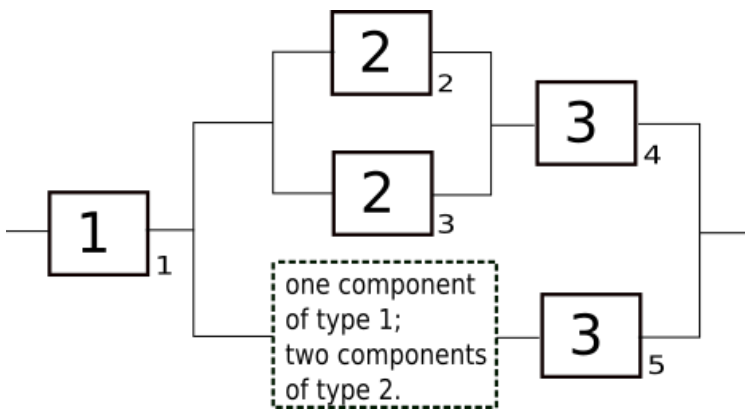

Figure 12: Grey system composed by eight components of 5 types with imprecision of the exact system configuration.

simulation methods are also directly applicable. Table B.7 shows the imprecise structural signature. For instance, if 2 components of type 1 and 1 component of type 3 are working the system can be either in a failing state or working with a probability of 0.5 (if the unknown part of the system is composed by an additional component of type 1). Since the system in Figure 12 is a coherent system, Algorithm 2 is used to estimated the bounds of the survival function by collecting the bound values (i.e. intervals) of the survival signature during the Monte Carlo simulation. In other words, the failure times of the components are sampled using the bounds of the failure time distribution as shown in the previous Section. In addition, in the Step 5' of Algorithm 2, the values of the survival signatures $\underline{\Phi_{t_{i}}}$ and $\overline{\Phi_{t_{i}}}$ are evaluated and in the Step 6' their values collected in two counters $\underline{V r(t)}=\underline{V r(t)}+\underline{\Phi_{t_{i}}}$ and $\overline{V r(j)}=\overline{V r(j)}+\overline{\Phi_{t_{i}}}, \forall j: j \cdot d t<t_{i}$. Hence, no additional Monte Carlo simulation are required to estimate the bounds of the survival function (the system reliability). If the component failure times are not affected by 
Table 3: Components failure types and distribution parameters for system of Fig.12

\begin{tabular}{lll}
\hline Component type & Distribution & Parameters \\
\hline 1 & Weibull (scale,shape) & $([1.6,1.8],[3.3,3.9])$ \\
2 & Exponential $(\lambda)$ & $([2.1,2.5])$ \\
3 & Weibull (scale,shape) & $([3.1,3.3],[2.3,2.7])$ \\
\hline
\end{tabular}

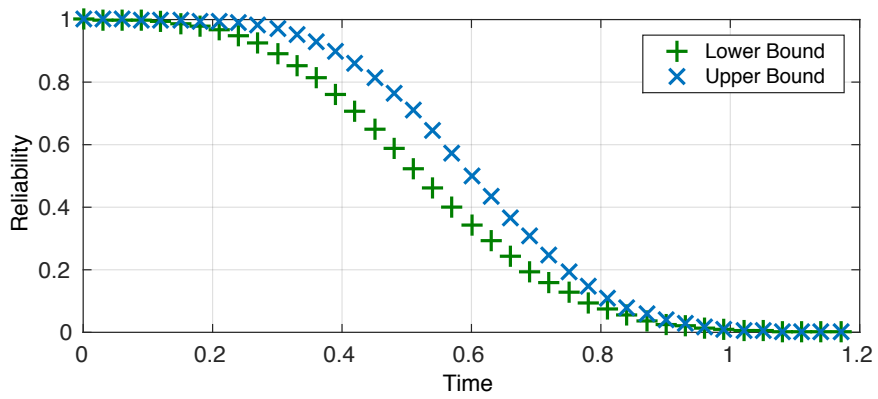

Figure 13: Upper and lower bounds of survival function for the system in Fig 12.

imprecision, only one Monte Carlo simulation would have been required to analyse the system with imprecision in the survival signature. In principle, Algorithm 1 can also be used for the estimation of the reliability bounds although it requires some modifications in the sampling of the system status.

The upper and lower bounds of survival function for the system with imprecision both in the survival signature and on the component distribution parameters are shown in Figure 13. The simulations have been performed using 5000 samples. This example shows the flexibility and the applicability of the simulation approaches proposed for the analysing of a systems affected by imprecision where no analytical solutions are available.

\subsection{Complex System}

In order to illustrate the efficiency and the applicability of the proposed simulation approaches, a complex system composed by 14 repairable components of 6 different types is analysed. The reliability block diagram of the system is shown in Figure 14 and the parameters of the components are reported in Table 4. The survival signature of this system can be referred in Appendix B.

First, the system is analysed without considering the repairs (i.e. transition

$2 \rightarrow 1$ is not allowed). Hence, the reliability of the system can be estimated adopting the proposed Algorithms 1 and 2. The results are shown in Figure 15 and Figure 16 for the case of non-repairable components with precise parameters and with imprecise parameters, respectively.

In case of repairable components or component with more than 1 allowed tran- 


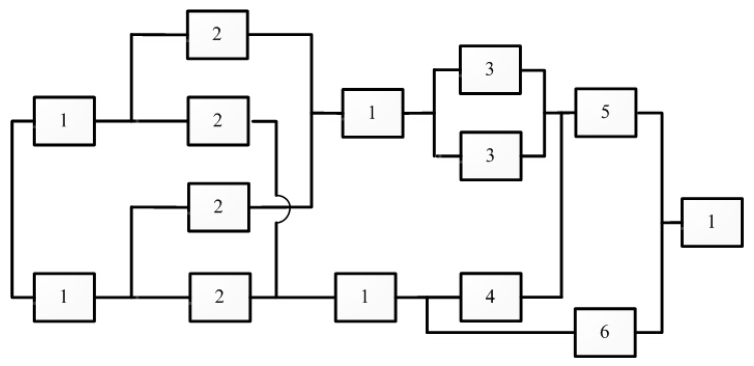

Figure 14: Reliability block diagram of the 16 component system.

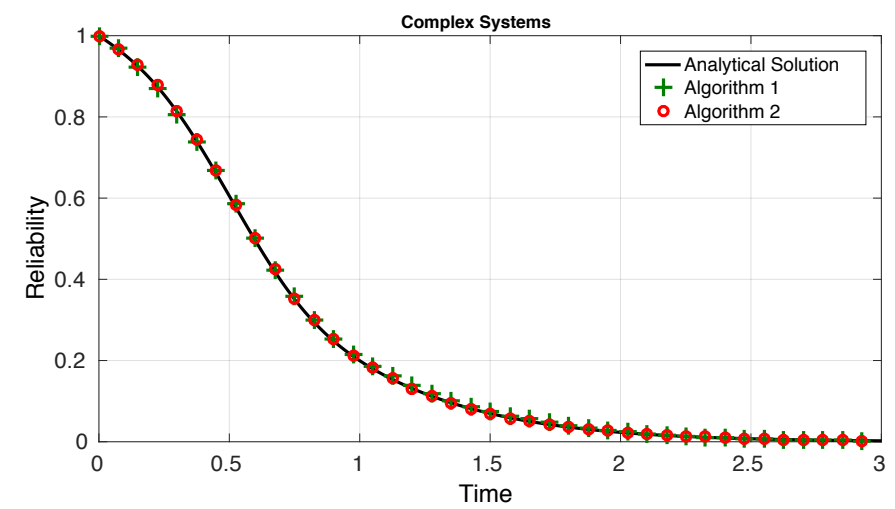

Figure 15: Survival function of the complex system calculated by Algorithms 1 and 2 and compared with analytical solution

sition, Algorithm 3 needs to be used. The proposed approach is generally applicable and allows to estimate the reliability of complex system based on the survival function. Figure 17 shows the survival function for the case of repairable components. The black line shows the results when the parameters of the failure and repair distributions are precisely known.

When imprecisions are considered within repairable system, the bounds of the survival function can be estimated by means of only two simulations as shown in Figure 6. These analyses require the calculation of the cumulative distribution function $(\mathrm{CDF})$ bounds for component failure and repair, which are expressed as $[\underline{F}, \bar{F}]$ and $[\underline{R}, \bar{R}]$ respectively. Then, the lower bound of the survival function 445 is estimated by considering the upper bound of the failure distributions and the lower bound for the repair distributions $(\bar{F}, \underline{R})$ while the upper bound is obtained adopting the lower bound for the failure distribution and the upper bound for the repair distribution $(\underline{F}, \bar{R})$. The interval of the survival function can be seen in Figure 17. 


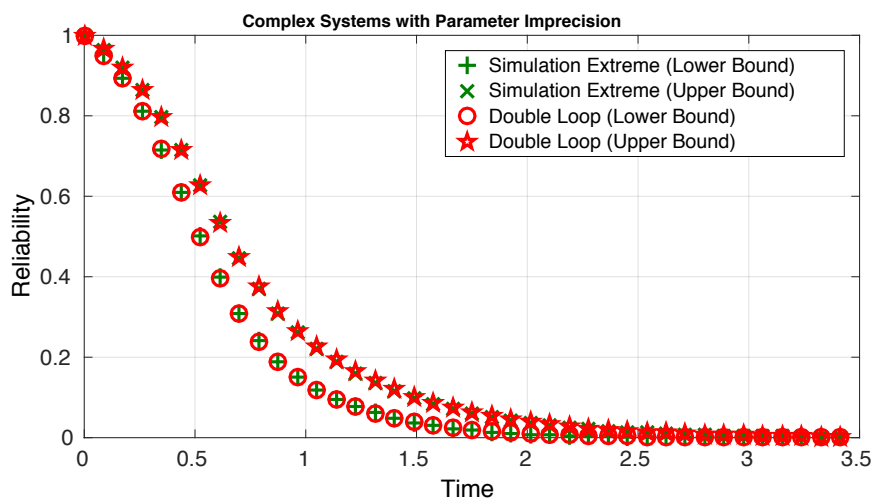

Figure 16: Bounds of survival function of the system with imprecision.

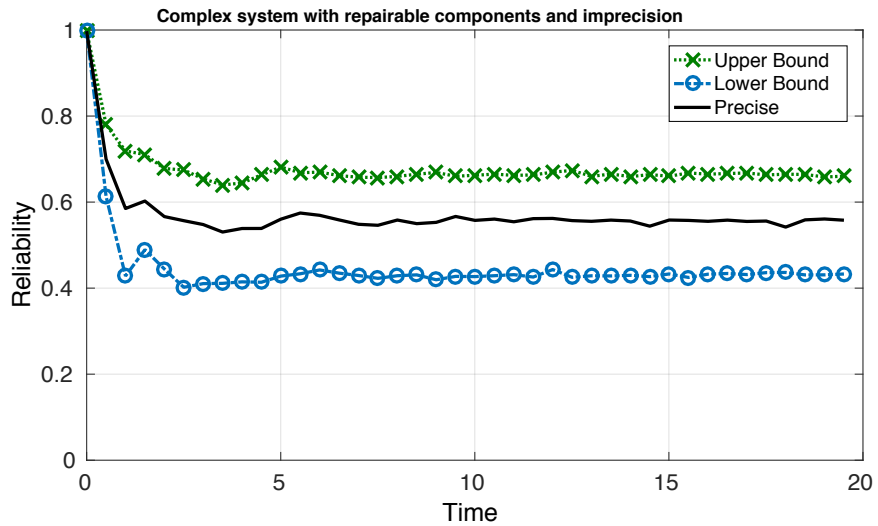

Figure 17: Survival function of the repairable complex system with imprecise and precise parameters, respectively. 
Table 4: Components failure (transition $1 \rightarrow 2$ ) and repair (transition $2 \rightarrow 1$ ) data for each component type of the complex system.

\begin{tabular}{|c|c|c|c|c|}
\hline $\begin{array}{l}\text { Component } \\
\text { type }(\mathrm{k})\end{array}$ & $\begin{array}{l}\text { Transition } \\
\text { (s) }\end{array}$ & Distribution & $\begin{array}{l}\text { Precise } \\
\text { parameters }\end{array}$ & $\begin{array}{l}\text { Imprecise } \\
\text { parameters }\end{array}$ \\
\hline 1 & $1 \rightarrow 2$ & $\begin{array}{l}\text { Exponential } \\
(\lambda)\end{array}$ & $(2.3)$ & $([2.1,2.5])$ \\
\hline 1 & $2 \rightarrow 1$ & $\begin{array}{l}\text { Uniform } \\
\text { (min,max) }\end{array}$ & $(0.4,0.6)$ & $([0.3,0.5],[0.5,0.7])$ \\
\hline 2 & $1 \rightarrow 2$ & $\begin{array}{l}\text { Exponential } \\
(\lambda)\end{array}$ & $(1.2)$ & $([0.9,1.4])$ \\
\hline 2 & $2 \rightarrow 1$ & $\begin{array}{l}\text { Uniform } \\
\text { (min,max) }\end{array}$ & $(0.9,1.1)$ & $([0.8,1.0],[1.0,1.2])$ \\
\hline 3 & $1 \rightarrow 2$ & $\begin{array}{l}\text { Weibull } \\
\text { (scale,shape) }\end{array}$ & $(1.7,3.6)$ & $([1.6,1.8],[3.3,3.9])$ \\
\hline 3 & $2 \rightarrow 1$ & Uniform & $(0.6,0.8)$ & $([0.5,0.7],[0.7,0.9])$ \\
\hline 4 & $1 \rightarrow 2$ & $\begin{array}{l}\text { Lognormal } \\
(\mu, \sigma)\end{array}$ & $(1.5,2.6)$ & $([1.3,1.8],[2.3,2.9])$ \\
\hline 4 & $2 \rightarrow 1$ & $\begin{array}{l}\text { Uniform } \\
\text { (min,max) }\end{array}$ & $(1.0,1.2)$ & $([0.9,1.1],[1.1,1.3])$ \\
\hline 5 & $1 \rightarrow 2$ & $\begin{array}{l}\text { Weibull } \\
\text { (scale,shape) }\end{array}$ & $(3.2,2.5)$ & $([3.1,3.3],[2.3,2.7])$ \\
\hline 5 & $2 \rightarrow 1$ & $\begin{array}{l}\text { Uniform } \\
(\min , \max )\end{array}$ & $(1.2,1.4)$ & $([1.1,1.3],[1.3,1.5])$ \\
\hline 6 & $1 \rightarrow 2$ & $\begin{array}{l}\text { Gamma } \\
\text { (scale,shape) }\end{array}$ & $(3.1,1.5)$ & $([2.9,3.3],[1.3,1.8])$ \\
\hline 6 & $2 \rightarrow 1$ & $\begin{array}{l}\text { Uniform } \\
(\min , \max )\end{array}$ & $(1.1,1.3)$ & $([1.0,1.2],[1.2,1.4])$ \\
\hline
\end{tabular}

In terms of computational effort of the analysis, the calculation of the survival signature of the complex system using the R-package ReliabilityTheory requires only a few seconds on a common desktop computer.

The performance for very large numbers of components is an important topic for future research where it is important to separate computation of survival signature from the simulation of the system. The first part can already be done for quite substantial systems using the approach proposed in [10,34] but which remains also a topic for research. The simulation of the system given the survival signature is almost independent on the number of components. In fact, the only parts of the simulation approach that scale with the number of components are the Steps 1 and 2 of the algorithm. In these steps the failure time of components is sampled and then sorted. However, the computational cost of this part is negligible (fraction of seconds) up to million of components as shown in Figure 18. The figure shows the computational cost of sampling the failure time of components 


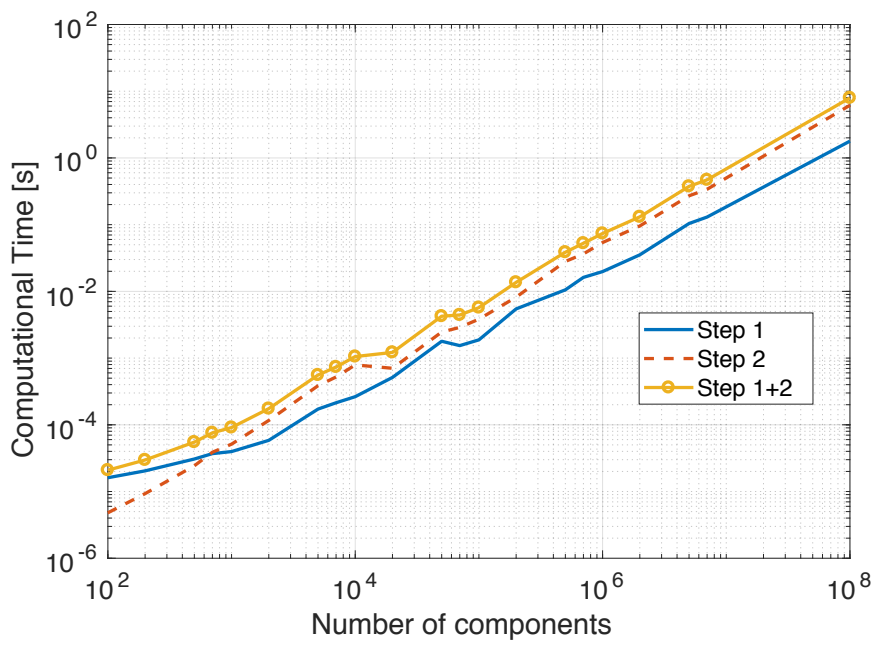

Figure 18: Scalability of the simulation algorithm with respect the number of components (Steps 1 and 2 only).

from exponential distribution (Step 1 of the Algorithm) and then sorting all the sampled times (Step 2). Steps 3-7 depend on the reliability of the components and the time of interest $\left(T_{f}\right)$ and scale linearly with the number of failures occurring before the time of interest. Clearly the total simulation time depends on the number of samples used. For the examples presented, the proposed simulation methods allow to estimate the survival function in less than 20 seconds using 5000 samples on a common desktop.

\section{Conclusions}

The survival signature has been shown to be a practical method for performing reliability analysis of complex systems with multiple component types. However, analytical methods are applicable only in few cases or adopting different levels of simplifications and assumptions.

In this paper, efficient simulation methods have been proposed for system reliability analysis. The methods proposed are based on survival signature, which need to be computed only once making the analysis very efficient. The proposed simulation methods are generally applicable and they can be used to analyse realistic and complex systems with non-repairable and repairable components. Recently, a case has been made for allowing the structure function for system reliability to be a, possibly imprecise, probability instead of a deterministic binary function [6]. This has advantages of more realistic reflections of uncertainty on system functioning and opens many interesting research questions. Such more general probabilistic structure functions can also be used in the survival signature in a straightforward 
manner, hence the simulation methods presented in this paper can also directly be applied.

The feasibility and effectiveness of the presented approaches have been illustrated with two numerical examples, the results indicate that simulation methods based on survival signature are efficient for analysing reliability on complex systems.

\section{Acknowledgement}

This work was partially supported by the UK Engineering and Physical Sciences Research Council (EPSRC) grant EP/M018709/1.

495 Geng Feng would like to thank the support of China Scholarship Council.

\section{Appendix A. Algorithms}

The Algorithms 1-3 of the proposed methods are shown in this appendix. In the Algorithms the letter $\mathrm{V}$ is used to represent vectors while the letter $\mathrm{M}$ represents matrices. The symbol $\sim$ is used represents sampling from given distribution.

\section{Appendix B. Survival Signature}

The tables in this appendix show the survival signature of the complex system of Figure 14. The rows with survival signature values equal to either 1 or 0 have been omitted. 


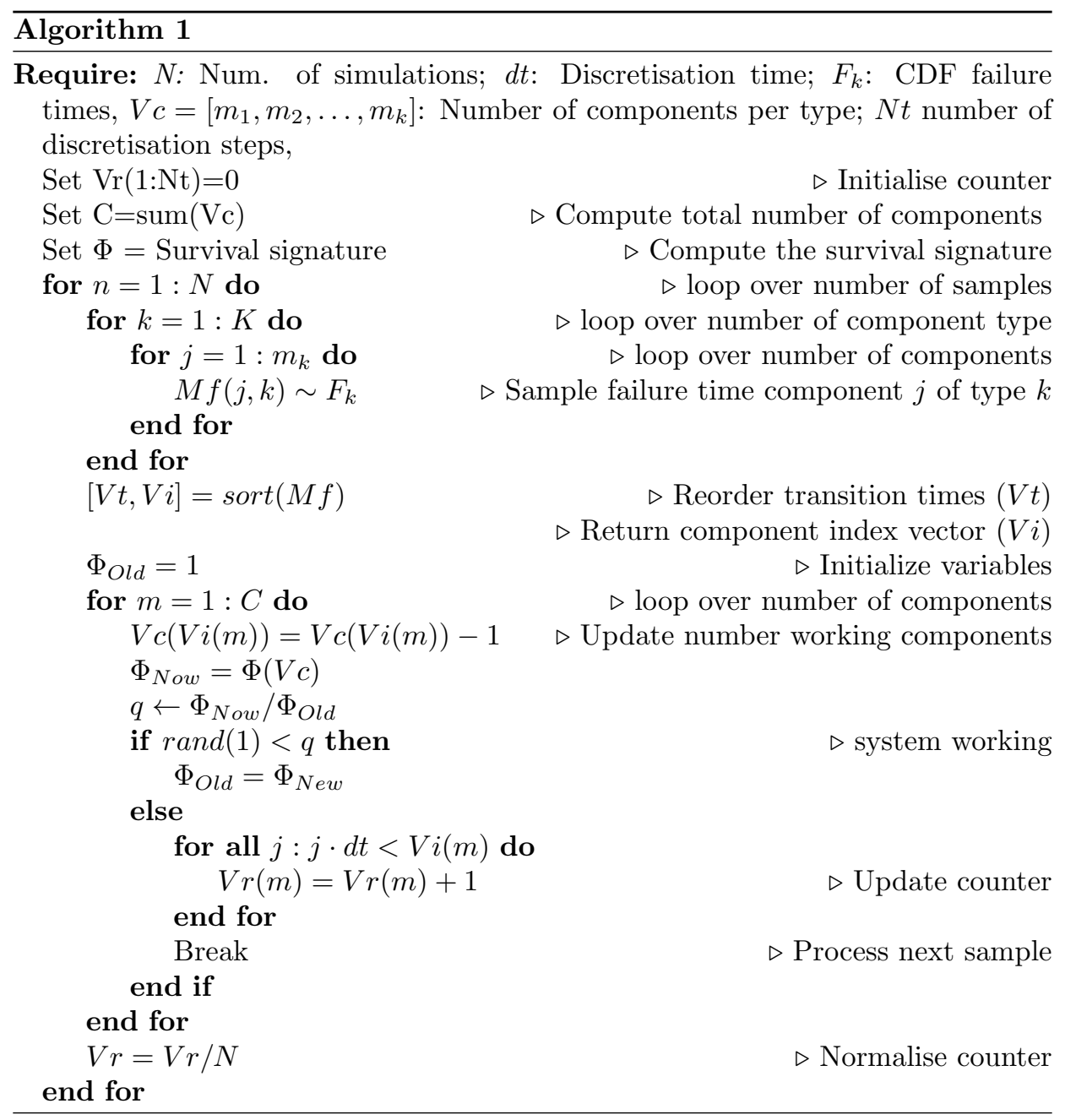




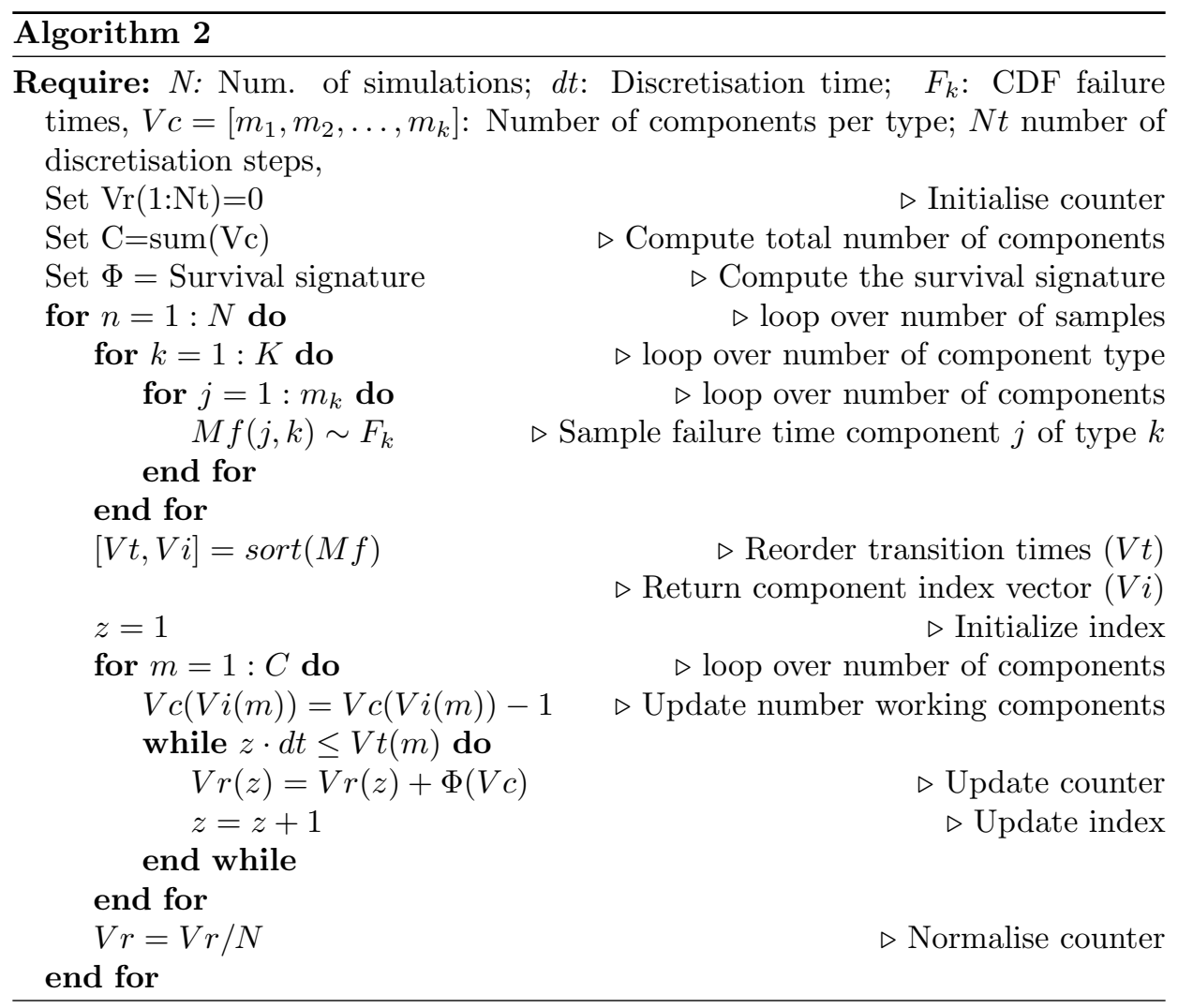




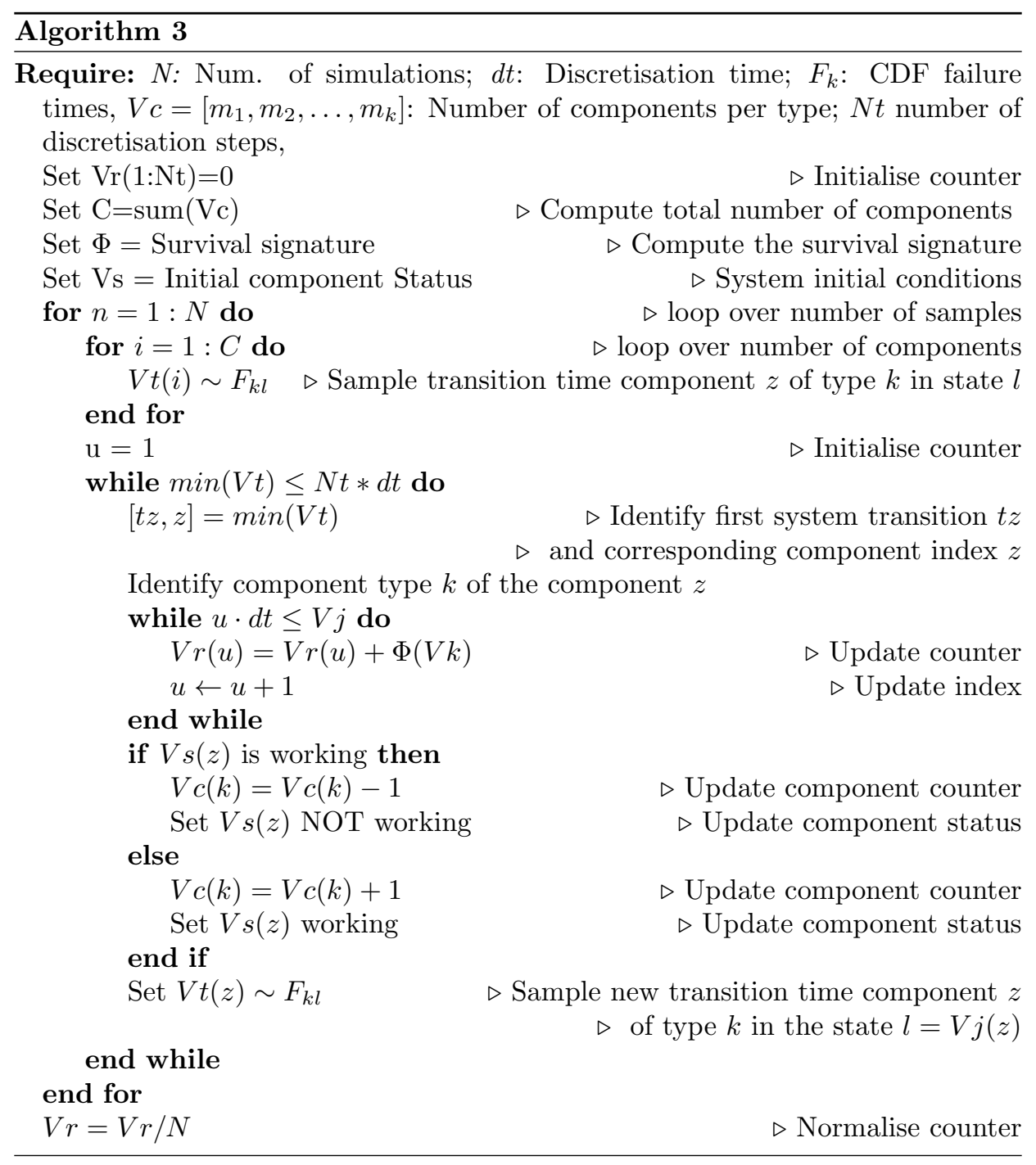


Table B.5: Survival signature of a complex system in Figure 14 ; rows with $\Phi\left(l_{1}, l_{2}, l_{3}, l_{4}, l_{5}, l_{6}\right)=$ 0 and $\Phi\left(l_{1}, l_{2}, l_{3}, l_{4}, l_{5}, l_{6}\right)=1$ are omitted

\begin{tabular}{lllllll}
\hline$l_{1}$ & $l_{2}$ & $l_{3}$ & $l_{4}$ & $l_{5}$ & $l_{6}$ & $\Phi\left(l_{1}, l_{2}, l_{3}, l_{4}, l_{5}, l_{6}\right)$ \\
\hline 3 & 1 & 0 & {$[0,1]$} & {$[0,1]$} & 1 & $1 / 20$ \\
3 & 1 & 0 & 1 & 1 & 0 & $1 / 20$ \\
3 & 1 & 1 & 0 & {$[0,1]$} & 1 & $1 / 20$ \\
3 & 1 & 1 & 1 & 0 & 1 & $1 / 20$ \\
3 & 1 & 2 & {$[0,1]$} & 0 & 1 & $1 / 20$ \\
3 & 1 & 2 & 0 & 1 & 0 & $1 / 20$ \\
3 & 1 & 1 & {$[0,1]$} & 1 & 1 & $1 / 10$ \\
3 & 1 & 1 & 1 & 1 & 0 & $1 / 10$ \\
3 & 1 & 2 & 0 & 1 & 1 & $1 / 10$ \\
3 & 1 & 2 & 1 & 1 & {$[0,1]$} & $1 / 10$ \\
3 & 2 & 0 & 0 & {$[0,1]$} & 1 & $1 / 10$ \\
3 & 2 & 0 & 1 & 0 & 1 & $1 / 10$ \\
3 & 2 & 0 & 1 & 1 & {$[0,1]$} & $1 / 10$ \\
3 & 2 & 1 & {$[0,1]$} & 0 & 1 & $1 / 10$ \\
3 & 2 & 1 & 0 & 1 & 0 & $1 / 10$ \\
3 & 2 & 2 & {$[0,1]$} & 0 & 1 & $1 / 10$ \\
3 & 2 & 2 & 0 & 1 & 0 & $1 / 10$ \\
3 & 3 & 0 & {$[0,1]$} & {$[0,1]$} & 1 & $3 / 20$ \\
3 & 3 & 0 & 1 & 1 & 0 & $3 / 20$ \\
3 & 3 & {$[1,2]$} & {$[0,1]$} & 0 & 1 & $3 / 20$ \\
3 & 3 & 1 & 0 & 1 & 0 & $3 / 20$ \\
3 & 3 & 2 & 0 & 1 & 0 & $3 / 20$ \\
3 & 4 & 0 & {$[0,1]$} & {$[0,1]$} & 1 & $1 / 5$ \\
3 & 4 & 0 & 1 & 1 & 0 & $1 / 5$ \\
3 & 4 & {$[1,2]$} & {$[0,1]$} & 0 & 1 & $1 / 5$ \\
3 & 4 & 1 & 0 & 1 & 0 & $1 / 5$ \\
3 & 4 & 2 & 0 & 1 & 0 & $1 / 5$ \\
4 & 1 & 0 & {$[0,1]$} & {$[0,1]$} & 1 & $1 / 5$ \\
\hline & & & & & &
\end{tabular}


Table B.6: Survival signature of a complex system in Figure 14 ; rows with $\Phi\left(l_{1}, l_{2}, l_{3}, l_{4}, l_{5}, l_{6}\right)=$ 0 and $\Phi\left(l_{1}, l_{2}, l_{3}, l_{4}, l_{5}, l_{6}\right)=1$ are omitted

\begin{tabular}{lllllll}
\hline$l_{1}$ & $l_{2}$ & $l_{3}$ & $l_{4}$ & $l_{5}$ & $l_{6}$ & $\Phi\left(l_{1}, l_{2}, l_{3}, l_{4}, l_{5}, l_{6}\right)$ \\
\hline 4 & 1 & 0 & 1 & 1 & 0 & $1 / 5$ \\
4 & 1 & {$[1,2]$} & {$[0,1]$} & 0 & 1 & $1 / 5$ \\
4 & 1 & 1 & 0 & 1 & 0 & $1 / 5$ \\
4 & 1 & 2 & 0 & 1 & 0 & $1 / 5$ \\
3 & 2 & {$[1,2]$} & {$[0,1]$} & 1 & 1 & $4 / 15$ \\
3 & 2 & 1 & 1 & 1 & 0 & $4 / 15$ \\
3 & 2 & 2 & 1 & 1 & 0 & $4 / 15$ \\
4 & 2 & {$[0,1,2]$} & {$[0,1]$} & 0 & 1 & $11 / 30$ \\
4 & 2 & 0 & 0 & 1 & 1 & $11 / 30$ \\
4 & 2 & 0 & 1 & 1 & {$[0,1]$} & $11 / 30$ \\
4 & 2 & 1 & 0 & 1 & 0 & $11 / 30$ \\
4 & 2 & 2 & 0 & 1 & 0 & $11 / 30$ \\
3 & {$[3,4]$} & 1 & {$[0,1]$} & 1 & 1 & $2 / 5$ \\
3 & 3 & 1 & 1 & 1 & 0 & $2 / 5$ \\
3 & 3 & 2 & 0 & 1 & 1 & $2 / 5$ \\
3 & 3 & 2 & 1 & 1 & {$[0,1]$} & $2 / 5$ \\
3 & 4 & 1 & 1 & 1 & 0 & $2 / 5$ \\
3 & 4 & 2 & {$[0,1]$} & 1 & 1 & $2 / 5$ \\
3 & 4 & 2 & 1 & 1 & 0 & $2 / 5$ \\
4 & 1 & {$[1,2]$} & {$[0,1]$} & 1 & 1 & $2 / 5$ \\
4 & 1 & {$[1,2]$} & 1 & 1 & 0 & $2 / 5$ \\
4 & 3 & 0 & {$[0,1]$} & {$[0,1]$} & 1 & $1 / 2$ \\
4 & 3 & 0 & 1 & 1 & 0 & $1 / 2$ \\
4 & 3 & {$[1,2]$} & {$[0,1]$} & 0 & 1 & $1 / 2$ \\
4 & 3 & 1 & 0 & 1 & 0 & $1 / 2$ \\
4 & 3 & 2 & 0 & 1 & 0 & $1 / 2$ \\
5 & 1 & 0 & {$[0,1]$} & {$[0,1]$} & 1 & $1 / 2$ \\
5 & 1 & 0 & 1 & 1 & 0 & $1 / 2$ \\
\hline & & & & & &
\end{tabular}


Table B.7: Imprecise survival signature of the system of Fig.12, $\phi\left(l_{1}, l_{2}, l_{3}\right)=0$ and $\phi\left(l_{1}, l_{2}, l_{3}\right)=$ 1 for both lower and upper bounds are omitted.

\begin{tabular}{llll}
\hline$l_{1}$ & $l_{2}$ & $l_{3}$ & {$\left[\phi\left(l_{1}, l_{2}, l_{3}\right)\right]$} \\
\hline 1 & 1 & 1 & {$[1 / 8,1 / 8]$} \\
1 & 1 & 2 & {$[1 / 4,1 / 4]$} \\
1 & 2 & 1 & {$[1 / 5,1 / 4]$} \\
1 & 2 & 2 & {$[3 / 7,1 / 2]$} \\
1 & 3 & 1 & {$[1 / 4,3 / 8]$} \\
1 & 3 & 2 & {$[1 / 2,1 / 2]$} \\
1 & 4 & 1 & {$[1 / 4,1 / 2]$} \\
1 & 4 & 2 & {$[1 / 2,1 / 2]$} \\
2 & 0 & 1 & {$[0,1 / 2]$} \\
2 & 0 & 2 & {$[0,1]$} \\
2 & 1 & 1 & {$[1 / 4,3 / 4]$} \\
2 & 1 & 2 & {$[1 / 2,1]$} \\
2 & 2 & 1 & {$[1 / 2,1]$} \\
2 & 3 & 1 & {$[3 / 4,1]$} \\
\hline
\end{tabular}

Table B.8: Survival signature of a complex system in Figure 14 ; rows with $\Phi\left(l_{1}, l_{2}, l_{3}, l_{4}, l_{5}, l_{6}\right)=$ 0 and $\Phi\left(l_{1}, l_{2}, l_{3}, l_{4}, l_{5}, l_{6}\right)=1$ are omitted

\begin{tabular}{lllllll}
\hline$l_{1}$ & $l_{2}$ & $l_{3}$ & $l_{4}$ & $l_{5}$ & $l_{6}$ & $\Phi\left(l_{1}, l_{2}, l_{3}, l_{4}, l_{5}, l_{6}\right)$ \\
\hline 5 & 1 & {$[1,2]$} & {$[0,1]$} & 0 & 1 & $1 / 2$ \\
5 & 1 & 1 & 0 & 1 & 0 & $1 / 2$ \\
5 & 1 & 2 & 0 & 1 & 0 & $1 / 2$ \\
4 & 4 & 0 & {$[0,1]$} & {$[0,1]$} & 1 & $3 / 5$ \\
4 & 4 & 0 & 1 & 1 & 0 & $3 / 5$ \\
4 & 4 & {$[1,2]$} & {$[0,1]$} & 0 & 1 & $3 / 5$ \\
4 & 4 & 1 & 0 & 1 & 0 & $3 / 5$ \\
4 & 4 & 2 & 0 & 1 & 0 & $3 / 5$ \\
4 & 2 & {$[1,2]$} & {$[0,1]$} & 1 & 1 & $2 / 3$ \\
4 & 2 & 1 & 1 & 1 & 0 & $2 / 3$ \\
4 & 2 & 2 & 1 & 1 & 0 & $2 / 3$ \\
4 & 3 & {$[1,2]$} & {$[0,1]$} & 1 & 1 & $4 / 5$ \\
4 & 3 & 1 & 1 & 1 & 0 & $4 / 5$ \\
4 & 3 & 2 & 1 & 1 & 0 & $4 / 5$ \\
4 & 4 & {$[1,2]$} & {$[0,1]$} & 1 & 1 & $4 / 5$ \\
4 & 4 & 1 & 1 & 1 & 0 & $4 / 5$ \\
4 & 4 & 2 & 1 & 1 & 0 & $4 / 5$ \\
5 & 2 & 0 & {$[0,1]$} & {$[0,1]$} & 1 & $5 / 6$ \\
5 & 2 & 0 & 1 & 1 & 0 & $5 / 6$ \\
5 & 2 & {$[1,2]$} & {$[0,1]$} & 0 & 1 & $5 / 6$ \\
5 & 2 & {$[1,2]$} & 0 & 1 & 0 & $5 / 6$ \\
\hline
\end{tabular}




\section{References}

[1] K. Kołowrocki, B. Kwiatuszewska-Sarnecka, Reliability and risk analysis of large systems with ageing components, Reliability Engineering \& System Safety 93 (12) (2008) 1821-1829.

[2] M. Modarres, What every engineer should know about reliability and risk analysis, Vol. 30, CRC Press, 1992.

[3] F. J. Samaniego, System signatures and their applications in engineering reliability, Vol. 110, Springer Science \& Business Media, 2007.

[4] S. Eryilmaz, Review of recent advances in reliability of consecutive k-out-of-n and related systems, Proceedings of the Institution of Mechanical Engineers, Part O: Journal of Risk and Reliability 224 (3) (2010) 225-237.

[5] F. P.A. Coolen, T. Coolen-Maturi, Generalizing the signature to systems with multiple types of components, in: Complex Systems and Dependability, Springer, 2012, pp. 115-130.

[6] F. P. Coolen, T. Coolen-Maturi, The structure function for system reliability as predictive (imprecise) probability., Reliability Engineering and System Safety (154) (2016) 180-187.

${ }_{520}$ [7] F. P.A. Coolen, T. Coolen-Maturi, A. H. Al-Nefaiee, Nonparametric predictive inference for system reliability using the survival signature, Proceedings of the Institution of Mechanical Engineers, Part O: Journal of Risk and Reliability 228 (5) (2014) 437-448.

[8] L. J.M. Aslett, F. P.A. Coolen, S. P. Wilson, Bayesian inference for reliability of systems and networks using the survival signature, Risk Analysis 35 (3) (2015) 1640-1651.

[9] G. Feng, E. Patelli, M. Beer, F. P. Coolen, Imprecise system reliability and component importance based on survival signature, Reliability Engineering \& System Safety 150 (2016) 116-125.

[10] S. Reed, An efficient algorithm for exact computation of system and survival signatures using binary decision diagrams, Reliability Engineering \& System Safety 165 (2017) 257-267. doi:10.1016/j.ress.2017.03.036.

URL http://dx.doi.org/10.1016/j.ress.2017.03.036

[11] P. R. Adduri, R. C. Penmetsa, System reliability analysis for mixed uncertain variables, Structural Safety 31 (5) (2009) 375-382.

[12] T. Augustin, F. P.A. Coolen, G. de Cooman, M. C.M. Troffaes, Introduction to imprecise probabilities, John Wiley \& Sons, 2014.

[13] J. Butler, J. Jia, J. Dyer, Simulation techniques for the sensitivity analysis of multi-criteria decision models, European Journal of Operational Research 103 (3) (1997) 531-546.

[14] R. Y. Rubinstein, Optimization of computer simulation models with rare events, European Journal of Operational Research 99 (1) (1997) 89-112.

[15] S. H. Zanakis, A. Solomon, N. Wishart, S. Dublish, Multi-attribute decision making: A simulation comparison of select methods, European journal of operational research 107 (3) (1998) 507-529.

[16] E. Zio, P. Baraldi, E. Patelli, Assessment of the availability of an offshore installation by monte carlo simulation, International Journal of Pressure Vessels and Piping 83 (4) (2006) 312-320. 
[17] H. George-Williams, E. Patelli, A hybrid load flow and event driven simulation approach to multi-state system reliability evaluation, Reliability Engineering \& System Safety 152 (2016) 351367. doi:10.1016/j.ress.2016.04.002.

[18] J. Song, W.-H. Kang, System reliability and sensitivity under statistical dependence by matrix-based system reliability method, Structural Safety 31 (2) (2009) 148 - 156, risk Acceptance and Risk Communication, Risk Acceptance and Risk Communication. doi:DOI:10.1016/j.strusafe.2008.06.012.

URL http://www.sciencedirect.com/science/article/ B6V54-4T6KF7K-2/2/a3c60ec860317e6a429b905d0f00f69c

[19] L. J.M. Aslett, Reliabilitytheory: Tools for structural reliability analysis. $\mathrm{r}$ package (2012). URL www.louisaslett.com

[20] L. J.M. Aslett, MCMC for inference on phase-type and masked system lifetime models, Ph.D. thesis, Trinity College Dublin (2012).

[21] E. Patelli, Handbook of Uncertainty Quantification, Springer International Publishing, Cham, 2016, Ch. COSSAN: A Multidisciplinary Software Suite for Uncertainty Quantification and Risk Management, pp. 1-69. doi:10. 1007/978-3-319-11259-6_59-1. URL http://dx.doi.org/10.1007/978-3-319-11259-6_59-1

[22] E. Patelli, M. Broggi, M. de Angelis, M. Beer, OpenCossan: An efficient open tool for dealing with epistemic and aleatory uncertainties, in: Vulnerability, Uncertainty, and Risk Quantification, Mitigation, and Management, ASCE, 2014, pp. 2564-2573.

[23] M. Marseguerra, E. Zio, Basics of the Monte Carlo Method with Application to System Reliability, LiLoLe Publishing, Hagen/Germany, 2002, iSBN: 3934447-06-6.

[24] D. A. Alvarez, Infinite random sets and applications in uncertainty analysis, Ph.D. thesis, Arbeitsbereich für Technische Mathematik am Institut fr Grundlagen der Bauingenieurwissenschaften. Leopold-Franzens-Universität Innsbruck, Innsbruck, Austria, available at https://sites.google.com/ site/diegoandresalvarezmarin/RSthesis.pdf (2007).

[25] B. Möller, M. Beer, Fuzzy-randomness - uncertainty in civil engineering and computational mechanics, Springer-Verlag, 2004.

[26] M. Beer, S. Ferson, Imprecise probabilities-what can they add to engineering analyses?, Mechanical Systems and Signal Processing 37 (1-2) (2013) 1 - 3.

[27] S. Ferson, V. Kreinovich, L. Ginzburg, D. S. Myers, K. Sentz, Constructing probability boxes and Dempster-Shafer structures, Report SAND2002-4015, Sandia National Laboratories, Albuquerque, NM, available at http://www. ramas.com/unabridged.zip (January 2003).

[28] M. Beer, Y. Zhang, S. T. Quek, K. K. Phoon, Reliability analysis with scarce information: Comparing alternative approaches in a geotechnical engineering context, Structural Safety 41 (0) (2013) 1 - 10. doi:http://dx.doi.org/10.1016/j.strusafe.2012.10.003.

URL http://www.sciencedirect.com/science/article/pii/ S0167473012000689

[29] R. E. Moore, R. B. Kearfott, M. J. Cloud, Introduction to Interval Analy- 

2009.

[30] E. Patelli, D. A. Alvarez, M. Broggi, M. de Angelis, Uncertainty management in multidisciplinary design of critical safety systems, Journal of Aerospace Information Systems 12 (2015) 140-169. doi:10.2514/1. I010273.

[31] M. Beer, S. Ferson, V. Kreinovich, Imprecise probabilities in engineering analyses, Mechanical systems and signal processing 37 (1) (2013) 4-29.

[32] L. V. Utkin, F. P. Coolen, Imprecise reliability: an introductory overview, in: Computational intelligence in reliability engineering, Springer, 2007, pp. 261-306.

[33] S. Ferson, C. A. Joslyn, J. C. Helton, W. L. Oberkampf, K. Sentz, Summary from the epistemic uncertainty workshop: consensus amid diversity, Reliability Engineering \& System Safety 85 (1) (2004) 355-369.

[34] G. Feng, S. Reed, E. Patelli, M. Beer, F. P. Coolen, Efficient reliability and uncertainty assessment on lifeline networks and imprecise survival signature, in: 2nd ECCOMAS Thematic Conference on Uncertainty Quantification in Computational Sciences and Engineering, 2017. 